\title{
Kinetic studies of gas hydrate formation with low-dosage hydrate inhibitors
}

\author{
TANG CuiPing ${ }^{1,2}$, DAI XingXue ${ }^{1,2}$, DU JianWei ${ }^{1,2}$, LI DongLiang ${ }^{1,2}$, \\ ZANG XiaoYa ${ }^{1,2}$, YANG XiangYang ${ }^{1} \&$ LIANG DeQing ${ }^{1 *}$ \\ ${ }^{1}$ Guangzhou Institute of Energy Conversion, Key Laboratory of Renewable Energy and Gas Hydrate, \\ Chinese Academy of Sciences, Guangzhou 510640, China \\ ${ }^{2}$ Graduate University of the Chinese Academy of Sciences, Beijing 100039, China
}

Received December 16, 2009; accepted February 3, 2010

\begin{abstract}
Pipeline blockage by gas hydrates is a serious problem in the petroleum industry. Low-dosage inhibitors have been developed for its cost-effective and environmentally acceptable characteristics. In a 1.072-L reactor with methane, ethane and propane gas mixture under the pressure of about $8.5 \mathrm{MPa}$ at $4{ }^{\circ} \mathrm{C}$, hydrate formation was investigated with low-dosage hydrate inhibitors PVP and GHI1, the change of the compressibility factor and gas composition in the gas phase was analyzed, the gas contents in hydrates were compared with PVP and GHI1 added, and the inhibition mechanism of GHI1 was discussed. The results show that PVP and GHI1 could effectively inhibit the growth of gas hydrates but not nucleation. Under the experimental condition with PVP added, methane and ethane occupied the small cavities of the hydrate crystal unit and the ability of ethane entering into hydrate cavities was weaker than that of methane. GHI1 could effectively inhibit molecules which could more readily form hydrates. The ether and hydroxy group of diethylene glycol monobutyl ether have the responsibility for stronger inhibition ability of GHI1 than PVP.
\end{abstract}

gases, hydrate, low dosage hydrate inhibitors, formation time

\section{Introduction}

Gas hydrates are ice-like crystalline inclusion compounds that form at high pressure and low temperature conditions by hydrogen bonds of water molecules, with the assistance of gases such as methane, ethane, or propane. There are three common crystal structures for gas hydrates SI, SII and SH. The cell unit of SI hydrate contains 46 water molecules which form two small cages and six large cages. SII hydrate is composed of sixteen small cages and eight large cages formed by 136 water molecules per cell unit. For SH hydrate cell unit there are three different cages formed by 34 water molecules $[1,2]$.

*Corresponding author (email: liangdq@ms.giec.ac.cn)
Since Hammerschmidt reported the formation of hydrates in gas pipelines in 1934, the problem of hydrate blockage of flow channels has been of major significance to the energy industry [3]. The oil and gas industry has expanded into deepwater and frozen areas for exploration and production of oil and gas. Thus the problem of hydrate blockages has become more challenging. Conventional thermodynamic hydrate inhibitors such as methanol, glycol or electrolyte have been widely used. These inhibitors are effective, but large concentrations are required, which impacts the project profits [4]. Additives such as methanol are harmful to the environment.

Low dosage hydrate inhibitors (LDHIs) have been proposed as a new means for preventing hydrate blockage in flow channels [5]. LDHIs include anti-agglomerants (AAs) and kinetic hydrate inhibitors (KHIs). Neither of them could 
change the thermodynamic condition of gas hydrate formation, but they could inhibit and delay hydrate nucleation and growth. These inhibitors are added at a low concentration, often less than $1 \mathrm{wt} \%$ active concentration. Environmentally friendly LDHIs have been used in many fields due to its cost-effectiveness [6-10].

Development of LDHIs has been studied by many researchers [6, 11-15], but the mechanism still remains unclear. Hutter et al. [16] used small-angle neutron scattering and contrast variation techniques to characterize the polymer conformations of two non-ionic water soluble polymers, poly(ethylene oxide) and poly( $N$-vinyl-2-pyrollidone), and thought there was strong perturbation in the conformation of the inhibitor polymer upon crystallization of the hydrate. Kvamme et al. [17] investigated possible effects of kinetic hydrate inhibitors at the hydrate surface by molecular dynamics simulations. They found that PVPCAP had more favorable interaction properties with hydrate water and better properties as a kinetic hydrate inhibitor than PVP. A modified version of the PVP monomer, where a hydroxyl group was added to the ring, could increase the attachment to the hydrate surface further. Kelland et al. [18] suggested that most kinetic inhibitors were polymers with the alkylamide group, so the alkyl group could penetrate an open cavity and then the amide group hydrogen bonding to the hydrate surface via the carbonyl group locking the alkylamide to the surface. Makogon and Sloan [19] regarded inhibitor polymer side groups adsorbed to the hydrate crystal surface by hydrogen bonding. By adsorbing on the hydrate crystal, the polymer forced the crystal to grow around and between the polymer stands, with a small radius of crystal curvature. The inhibitors also sterically blocked methane from entering and completing a hydrate cavity. Gómez Gualdrón and Balbuena [20] performed classical molecular dynamics simulations of lathrate-methane-water and an inhibitor of clathrate formation and regarded at the initial stages of clathrate formation the hydrophobic inhibitors might block the surface of the nascent crystals, whereas the hydrophilic ones act mainly by disruption of the water structure. Jensen et al. [21] found addition of polyvinylpyrrolidone (PVP) to the aqueous phase could slightly reduce the gas dissolution rate, but the induction times were substantially prolonged upon addition of PVP. The prolongation of induction times according to the model was regarded to be due to a change in the nuclei-substrate contact angle. The latest data showed the presence of VC-713 lowered the interfacial tension, increased the concentration of methane on the surface of the aqueous phase, and thus was favorable for the nucleation of hydrates at the gas/liquid interface to some extent [22].

Those studies about the mechanism are based on structures of LDHIs mostly by molecular modeling. Investigation of the mechanism of LDHI with a synergist is rare, especially for the influence of the synergist. In this work we focused on guest molecules, with the main purpose of investigating the influence of different components of gas mixture on the inhibition ability of LDHIs and discussing the mechanism of GHI1, a LDHI with a synergist.

\section{Apparatus and experimental procedure}

The experimental apparatus includes the reaction cell, the temperature controller, temperature and pressure measure system and data acquisition system [12]. A cylindrical highpressure cell, made of stainless steel, was used to produce gas hydrates. A stainless-steel flange, which has appropriate ports for access to the interior, was used to seal the cell on the top. The cell was designed to operate at pressures up to $20 \mathrm{MPa}$ and temperatures in the range of $-15-50{ }^{\circ} \mathrm{C}$ with its available volume of $1072 \mathrm{~cm}^{3}$. The interlayer of the cell was cooled using circulated cooling water from the coolant bath. The cell was enclosed by insulation. A stirrer with a speed of $0-1250 \mathrm{r} / \mathrm{min}$ was used to agitate the liquid. Two platinum resistance thermometers were used to measure the experimental temperature with an accuracy of $\pm 0.1{ }^{\circ} \mathrm{C}$. One thermometer extended into the bottom of the cell, and the other extended into the gas phase at the top. The pressure of the cell was measured using a 10-MPa pressure sensor with an accuracy of $\pm 0.5 \%$. The data was logged by the Agilent 34901A. Components of gases were measured by HP6890 gas chromatogram.

A typical procedure was as follows. Approximately $198.0 \mathrm{~g}$ of the aqueous phase was injected into the cell for each test. The cell was subsequently pressurized by natural gas to a pressure less than 1.0 MPa. When the system was cooled to $4{ }^{\circ} \mathrm{C}$, the cell pressure was increased to the experimental pressure $8.5 \mathrm{MPa}$ over a span of 3-5 min into the cell. The gas input valve was turned off and the stirrer turned on.

In all the tests, the reactor liquid content was about 198.0 $\pm 1.0 \mathrm{~g}$. The cell was maintained at $4{ }^{\circ} \mathrm{C}$ and the pressure at 8.5 MPa. The estimated subcooling was at $14.7^{\circ} \mathrm{C}$ calculated by CSMHYD software. However, for the limit of pressure apparatus, the experimental pressure was kept at 8.5-9.0 MPa. The chemical concentration for all additives was $0.5 \mathrm{wt} \%$ based on distilled water mass.

The gas composition and chemicals used in experiments were as follows. Natural gas was composed by $3.05 \mathrm{vol} \%$ ropane, 5.00 vol\% ethane and methane balance from Fushan Kede Gas Co. Diethylene glycol monobutyl ether $\left(\mathrm{C}_{8} \mathrm{H}_{18} \mathrm{O}_{3}\right)$ was more than $99.0 \mathrm{wt} \%$ purity from Tianjin Bodi Chemicals Co., Ltd. PVP, Inhibex 100 and Inhibex 501 were kindly provided by ISP Asia Pacific Co., Ltd. In all the tests DI water was used.

\section{Results and discussion}

The point of $t=0$ is defined as the moment that the pressure reaches $8.5 \mathrm{MPa}$ and magnetic stirring begins. The formation time in this paper is a significant parameter and is regarded 
as time from zero to the moment of abruptly rising temperature. It is catastrophic hydrate formation time. Gas was supplied without pre-cooling.

A typical plot of pressure and temperature data in the cell for a test carried out using distilled water during the hydrate formation process is available in another paper [12]. GHI1 contained polyvinylpyrrolidone and diethylene glycol monobutyl ether in the weight ratio of $1: 1$.

\subsection{Process of gas hydrate formation}

Gas hydrate formation is expressed by the equation as follows:

$$
\mathrm{G}+n \mathrm{H}_{2} \mathrm{O} \rightarrow \mathrm{G} \cdot n \mathrm{H}_{2} \mathrm{O}
$$

Here $\mathrm{G}$ represents natural gas molecules, $n$ represents the mole of water when $1 \mathrm{~mol}$ gas is consumed, and $\mathrm{G} \cdot n \mathrm{H}_{2} \mathrm{O}$ represents gas hydrates. Formation of $\mathrm{G} \cdot n \mathrm{H}_{2} \mathrm{O}$ experienced these stages, from gas dissolution through metastable structures to stable gas hydrates, shown in Figure 1.

Gas hydrate formation can be divided into gas dissolution, hydrate nucleation and agglomeration stages as shown in Figure 1. Under the condition of gas hydrate formation, water molecules form quasi-cavities by hydrogen bonds. When gases dissolve, gas molecules are entrapped into these quasi-cavities to form labile clusters. These clusters could agglomerate together, which are in quasi-equilibrium with labile clusters until the species reach a critical radius to form stable hydrate nuclei. Finally the hydrates grow catastrophically when hydrate nuclei agglomerate. LDHIs could inhibit gas hydrate formation during nucleation and agglomeration stages.

\subsection{Formation time}

Formation time is a significant parameter in this investigation or for selecting inhibitors in the field. Time is not a good means to assess the inhibitors for low repetition, but in practice time is a significant factor which should be considered. Therefore, experiments were repeated at least four times, and each time the cell was cleaned and fresh fluid was added. Table 1 shows the exact formation time for each run of different inhibitors.

It seems the longer the formation time is, the worse the repeat is. The influence of disturbing conditions on the results would be more apparent with the experimental time increasing. In the aggregate, the repetition is good and time could distinguish among different inhibitors. The experimental result for commercial inhibitors of Inhibex 100, Inhibex 501 and PVP shows that the tendency of inhibition ability in the lab is the same as the effect in the field. Formation time could be the basis of the next analysis.

\subsection{Analysis of gas hydrate reactions with PVP}

PVP is one of the earliest kinetic inhibitors with a relatively simple structure and the main component of GHI1. First the gas hydrate reaction with PVP was analyzed.

The content of each gas component in the gas phase was detected by Gas Chromatography, and calculated by RedkichKwong-Soave equation. Gas contents in the gas phase and hydrates and compressibility factor $Z$ could be obtained. It is noteworthy that the speed of stirring decreases to prolong the reaction process in order to get enough formation time to investigate the whole reaction. Stirring speed is regarded not to change the mechanism of gas hydrate formation with

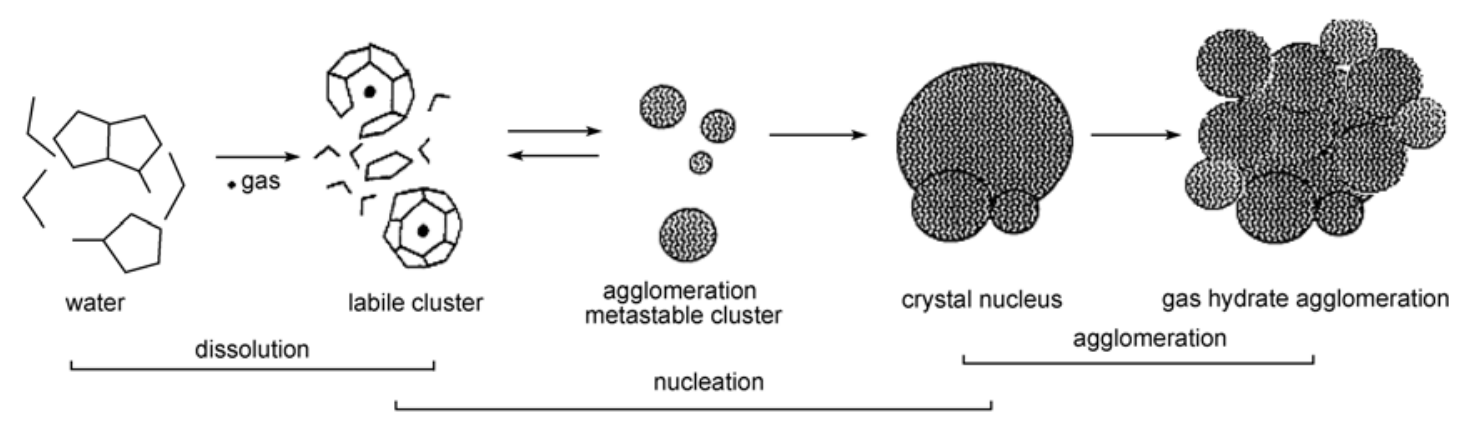

Figure 1 Gas hydrate formation process.

Table 1 Formation time with different inhibitors (min)

\begin{tabular}{|c|c|c|c|c|c|c|}
\hline Additives & Run 1 & Run 2 & Run 3 & Run 4 & Average & Standard errors \\
\hline PVP & 650 & 650 & 670 & 660 & 658 & 8 \\
\hline Inhibex 100 & 1140 & 1000 & 770 & 1060 & 993 & 138 \\
\hline Inhibex 501 & 1740 & 2500 & 1800 & 2200 & 2060 & 310 \\
\hline GHI1 & 4750 & 4530 & 6200 & 5500 & 5245 & 658 \\
\hline
\end{tabular}


LDHIs. In Figures 2 and 3 the gas hydrate formation time is about 800 min with PVP added.

Figure 2 shows compressibility factor $Z$ is high at first as gas dissolution has not reached the equilibrium state. With the stirrer beginning, the speed of gas dissolution increases and temperature and pressure decrease, which leads to decreasing of $Z$. When decreasing to some extent, $Z$ turns to increase. As temperature is stable at this time, the change of gas contents and pressure decreasing result in increase of $Z$. In other words, gases are consumed, so PVP cannot effectively inhibit gas hydrate nucleation, but hydrates have not formed catastrophically as there is no drastic temperature rising.

The change of a sample plot of gas phase components with time is shown in Figure 3. The contents of each component keep decreasing. This demonstrates that PVP cannot effectively inhibit gas hydrate nucleation. Comparison of three gas contents shows that of methane decreases more significantly than propane and ethane. This result may be caused by the much greater amount of methane than that of ethane and propane.

When natural gas hydrates are formed, the gas contents significantly decrease, especially for methane and propane, but for ethane the change is mild. Perhaps the crystal struc-



Figure 2 The change of compressibility factor with reaction time with $0.5 \mathrm{wt} \%$ PVP.

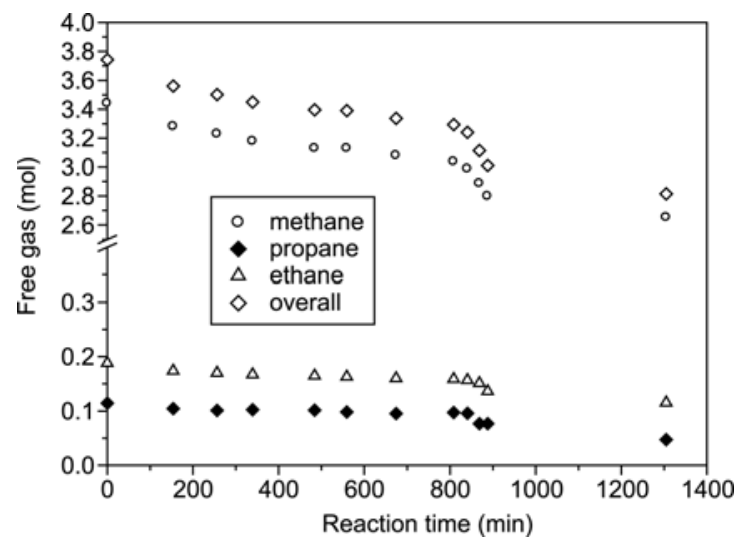

Figure 3 Gas contents in the gas phase with 0.5 wt $\%$ PVP added. ture of gas hydrate formed determines it. S II hydrates form under this condition, which have eight large cavities and sixteen small cavities shown in Figure 4. The average cavity radius is respectively $3.91 \AA$ and $4.73 \AA$ for the two kinds of cavities. According to natural gas components, the proportion of each component in gas hydrates is 0.6034:0.0299: 0.3667 calculated by CSMHYD software. In other words methane and ethane occupy the small cavities and propane occupies the large cavities in this condition. A ratio of lower bound of molecule diameter to cavity's diameter is about 0.76 , below which the molecular attractive force cannot contribute to cavity stability. Above the upper bound ratio of about 1.0, the guest molecule cannot fit into a cavity without distortion. The van der Waals diameter of ethane is $5.5 \AA$, and the ratio of molecule diameter to cavity's diameter is 1.10 . Therefore, ethane can not occupy small cavity unless distortion. The diameter of methane molecule is 4.36 with the ratio of molecule diameter to cavity's diameter being 0.868 , so the stable cavity could be obtained [1]. Methane molecules could apparently occupy small cavities prior to ethane, and have a much larger content, which leads to a little proportion in small cavities and mild decrease in Figure 3 for ethane at the catastrophic gas hydrate formation.

\subsection{Analysis of gas hydrate reaction with GHI1}

The gas sample in the run 4 in Table 1 with GHI1 was analyzed. The calculated method is the same as with PVP, the corresponding results are shown in Figures 5 and 6.

The curve change tendency is similar in Figure 5 to Figure 1. Therefore, neither GHI1 nor PVP could effectively inhibit gas hydrate nucleation. Z's change is smaller with GHI1 than PVP before gas hydrates' catastrophic formation, which implies that the inhibition ability of GHI1 is better than that of PVP.

Figure 6 shows contents of methane decrease more significantly than that of propane and ethane especially before the catastrophic growth in Figure 3. This result can be explained by two reasons: the amount of methane is much more than that of ethane and propane, and GHI may inhibit ethane and propane to form hydrates more effectively than methane. It is noteworthy that the gas hydrate formation time with GHI1 is much longer than that with PVP. When natural gas hydrates are formed, the gas contents significantly

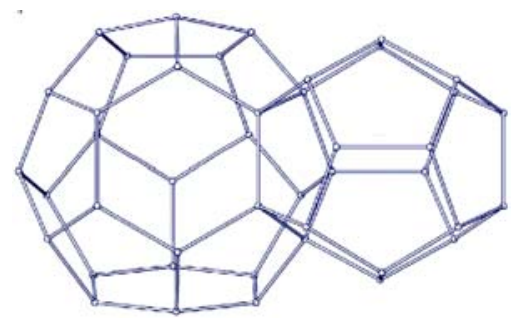

Figure 4 A view of the large and the small cavity of cubic structure II. 


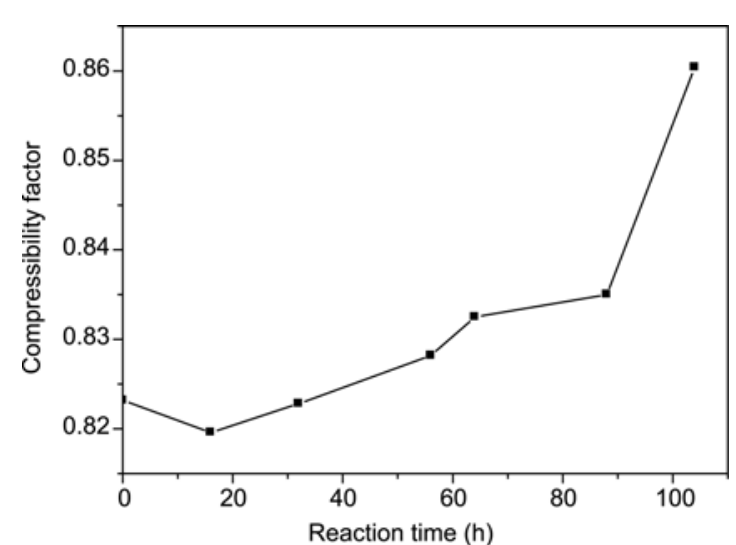

Figure 5 The change of compressibility factor with reaction time with $0.5 \mathrm{wt} \%$ GHI1.

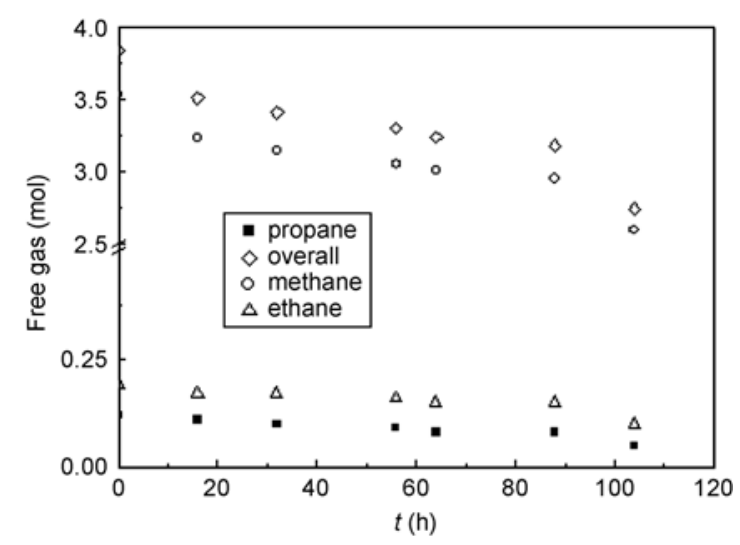

Figure 6 The plot of free gas versus time for the test with $0.5 \%$ GHI1.

decrease, so gases are consumed rapidly and gas hydrates form catastrophically. More ethane and propane are consumed after GHI1 loses its effect. Perhaps the contents which are favorable for hydrate formation could be inhibited by GHI more effectively than those which form hydrates with a severe condition.

Two samples are proposed at $88.8 \mathrm{~h}$ and $104.8 \mathrm{~h}$ and gas hydrates' catastrophic formation is at $91.6 \mathrm{~h}$. Components of gases are shown in Table 2. In the experiment more than $66 \%$ of the total propane and more than $47 \%$ ethane and $26 \%$ methane were consumed. Propane remains the most readily hydrated form among methane, ethane and propane. Therefore, except using inhibitors, to separate the components

Table 2 Components of gas data during reactions

\begin{tabular}{ccccccccc}
\hline & \multicolumn{3}{c}{ Mole in hydrates (mol) } & & \multicolumn{3}{c}{ Consumption (\%) } \\
\cline { 2 - 3 } \cline { 6 - 7 } \cline { 5 - 7 } & $t=0$ & $t=88.8 \mathrm{~h}$ & $t=104.8 \mathrm{~h}$ & & $t=0$ & $t=88.8 \mathrm{~h}$ & $t=104.8 \mathrm{~h}$ \\
\hline Methane & 0 & 0.58 & 0.94 & & 0 & 16.4 & 26.6 \\
Ethane & 0 & 0.04 & 0.09 & & 0 & 21.1 & 47.4 \\
Propane & 0 & 0.04 & 0.08 & & 0 & 33.3 & 66.7 \\
\hline
\end{tabular}

which readily form hydrates is a good method to avoid hydrate formation.

\subsection{Gas hydrate reaction degree}

It is challenging to compare the amount of hydrates at different formation time with GHI1 or PVP added. Therefore, reaction degree $D$ is suggested and defined as $D=t / t_{\mathrm{f}}$, where $t$ represents any time during the reaction and $t_{\mathrm{f}}$ is the formation time.

The change of gas contents in the hydrate phase with reaction degree is shown in Figure 7. The contents increase with reaction time, which is also shown in figures about $Z$. Because of different formation time the gas contents in hydrates with GHI1 are larger than that with PVP at the same reaction degree. It seems that GHI1 is similar to an antiagglomerant which is regarded as a kinetic inhibitor.

\subsection{Inhibition mechanism of GHI1}

The chemical structures of GHI1 and water are shown in Figure 8 , in which all oxygen and nitrogen atoms could form hydrogen bonds with hydrogen in water molecules. Hydrogen bonds could also be formed between water molecules, which are the basis of hydrate cavities. Gas hydrate formation is delayed by hydrogen bonds between PVP and the surface of cavities. The side alkylamide group penetrates an open cavity, and long chains as space resistance inhibit agglomeration of hydrate nuclei. Diethylene glycol monobutyl ether cannot occupy the hydrate cavities of S II for large van der Walls' radius. The ether and alcohol structure could help to form hydrogen bonds, especially hydroxyl groups which could form stronger hydrogen bonds as water.

Comparing PVP and GHI1 as LDHIs, it si suggested the difference of inhibition ability is caused by diethylene glycol monobutyl ether. Diethylene glycol monobutyl ether is water-soluble liquid and has not been used as a LDHI, but has the ability to form hydrogen bonds with water molecules.

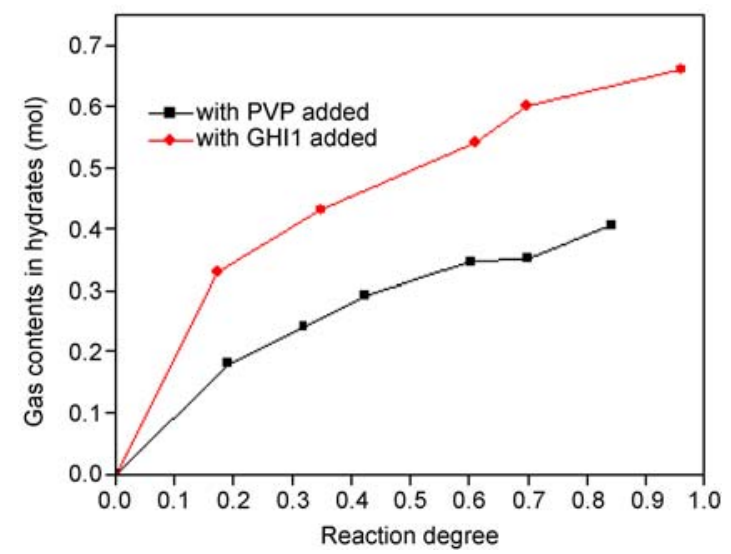

Figure 7 Gas contents in hydrates at different $D$ with GHI1 and PVP. 


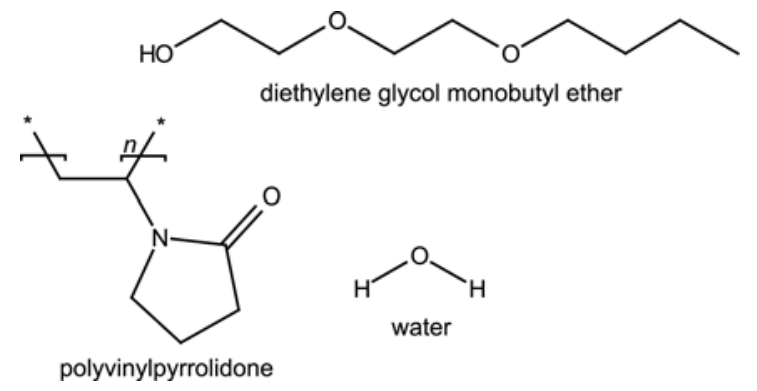

Figure 8 Chemical structures of GHI1 and water.

Whether the hydrogen bonds are strong depends on the electron cloud density of a lone pair of electrons and naked hydrogen. The hydrogen nucleus appears nearly naked from outside the water molecule. Cloud density of oxygen with a single bond is higher than a double bond. Therefore, hydrogen bonds between PVP and water molecules are weaker than diethylene glycol monobutyl ether and water. Diethylene glycol monobutyl ether may force gas hydrate formation to go across a high energy barrier through space resistance caused by strengthened hydrogen bonds, which is the basis of hydrate cavities and the key factor to inhibit hydrate formation.

\section{Conclusions}

In a high pressure cell at $14.7^{\circ} \mathrm{C}$ supercooling gas hydrate formation was investigated with inhibitors GHI1 and PVP. Results were obtained concerning the compressibility factor and gas distribution in gas and hydrate phases.

The change tendency of $Z$ is similar with GHI1 to PVP. Neither GHI1 nor PVP could effectively inhibit gas hydrate nucleation, but the inhibition ability of GHI1 is better than that of PVP.

According to the gas composition, structure II gas hydrates form at these tests. Through calculation it is regarded that methane and ethane occupy the small cavities and propane occupies the large cavities. Methane molecules could occupy small cavities prior to ethane.

Methane contents decrease more significantly than propane and ethane, especially before the catastrophic growth during the reaction. Perhaps the contents which are favorable for hydrate formation could be inhibited by GHI more effectively than those which form hydrates with a severe condition. This provided a means to separate methane, ethane and propane.

The difference of inhibition ability between GHI1 and PVP is caused by diethylene glycol monobutyl ether. The ether and alcohol structure could help to form hydrogen bonding with water molecules. Diethylene glycol monobutyl ether may force gas hydrate formation to go across a high energy barrier through space resistance caused by hydrogen bonding.
This work was supported by the National Natural Science Foundation of China (50906087).

1 Sloan ED. Clathrate Hydrate of Nature Gases. 2nd ed. New York: Marcel Dekker Inc., 1998, 27-49

2 Zang XY, Fan SS, Liang DQ, Li DL, Chen GJ. Influence of 3A molecular sieve to tetrahydrofuran(THF) hydrate formation. Sci China Ser B-Chem, 2008, 51: 893-900

3 Hammerschmidt EG. Formation of gas hydrates in natural gas transmission lines. Ind Eng Chem, 1934, 26: 851-855

4 Lovell D, Pakulski M. Hydrate inhibition in gas wells treated with two low dosage hydrate inhibitors. In: Proceedings of the SPE International Symposium on Gas Technology, Alberta, Canada, 2002, SPE 75668

5 Kelland MA, Svartaas TM, Dybvik L. Studies on gas hydrate inhibitors. In: Proceedings of the SPE Offshore Europe Conference. Aberdeen: SPE, 1995, 30420

6 Kelland MA. History of the development of low dosage hydrate inhibitors. Energy Fuels, 2006, 20: 825-847

7 Fu SB, Cenegy LM, Neff CS. A summary of successful field application of a kinetic hydrate inhibitor. In: Proceedings of the SPE International Symposium, Texas, USA, 2001, SPE 65022

8 Glénat P, Peytavy JL, Holland -Jones N, Grainger M. South-Pars phases 2 and 3: The kinetic hydrate inhibitor (KHI) experience applied at field start-up. In: Proceedings of the SPE International Symposium, Abu Dhabi, U.A.E, 2004, SPE 88751

9 Swanson TA, Petrie M, Sifferman TR. The successful use of both kinetic hydrate and paraffin inhibitors together in a deepwater pipeline with a high water cut in the Gulf of Mexico. In: Proceedings of the SPE International Symposium, Texas, USA, 2005, SPE 93158

10 Thieu V, Frostman LM. Use of low-dosage hydrate inhibitors in sour systems. In: Proceedings of the SPE International Symposium, Texas, USA, 2005, SPE 93450

11 Lederhos JP, Long JP, Sum A, Christiansen RL, Sloan ED. Effective kinetic inhibitors for natural gas hydrate. Chem Eng Sci, 1996, 51: 1221-1229

12 Tang CP, Du JW, Liang DQ, Fan SS, Li XS, Chen Y, Huang NS. Investigation on a new natural gas hydrate kinetic inhibitor (in Chinese). $J$ Xi'an Jiaotong Univ, 2008, 42: 333-336, 367

13 Karaaslan U, Parlaktuna M. PEO-a new hydrate inhibitor polymer. Energy Fuels, 2002, 16: 1387-1391

14 Kelland MA, Svartås TM, Andersen LD. Gas hydrate anti-agglomerant properties of polypropoxylates and some other demulsifiers. J Pet Sci Eng, 2009, 64: 1-10

15 Villano LD, Kelland MA. Tetrahydrofuran hydrate crystal growth inhibition by hyperbranched poly(ester amide)s. Chem Eng Sci, 2009, 64: 3197-3200

16 Hutter JL, King HE, Lin MY. Polyer conformations of gas hydrate kinetic inhibitors: a small angle neutron scattering study. J Chem Phys 2000, 112: 2523-2532

17 Kvamme B, Kuznetsova T, Aasoldsen K. Molecular dynamics simulations for selection of kinetic hydrate inhibitors. J Mol Graphics Modell, 2005, 23: 524-536

18 Kelland MA, Svartaas TM. A new class of kinetic hydrate inhibition. Ann N Y Acad Sci, 2000, 912: 281-293

19 Makogon TY, Sloan ED. Mechanism of kinetic hydrate inhibitors. In: Proceedings of 4th International Hydrate Conference, Yokohama: Keio University, 2002, 498-503

20 Gómez-Gualdrón DA, Balbuena PB. Classical molecular dynamics of clathrate-methane-water-kinetic inhibitor composite systems. J Phys Chem C, 2007, 111: 15554-15564

21 Jensen L, Thomsen K, von Solms N. Propane hydrate nucleation: Experimental investigation and correlation. Chem Eng Sci, 2008, 63: 3069-3080

22 Peng BZ, Sun CY, Liu P, Liu YT, Chen J, Chen GJ. Interfacial properties of methane/aqueous vc-713 solution under hydrate formation conditions. J Colloid Interface Sci, 2009, 336: 738-742 